\title{
Treatment of 76 Cases of Sinusitis with Acupuncture
}

TIAN Hai-ying (田海英), ZHAO Feng-jun (赵风军)

People's Hospital of Yanggu, Shandong 252300, P. R. China

\author{
【关键词】针刺疗法; 泻法; 鼻窦炎 \\ 【Key Words】 Acupuncture Therapy; Reducing Method; Sinusitis \\ 【CLC Number】 R246.8 \\ 【Document Code】B
}

Sinusitis is a prevalent nasal disease, characterized by profuse turbid nasal discharge, usually coupled with headache, nasal stuffiness, declining of smell, and also dizziness in protracted cases. The author treated 76 cases by acupuncture, and it was reported as follows.

\section{General Data}

Of all 76 subjects, 47 were male and 29 were female, aged from 3 to 64 years, with a duration ranging from $47 \mathrm{~d}$ to 3 years.

\section{Treatment Methods}

Acupoints: Bilateral Yingxiang (LI 20), Fengchi (GB 20), Qiuxu (GB 40), Yinlingquan (SP 9).

Operation: The patient took a supine position. After routine sterilization, the aforementioned acupoints were punctured by filiform needles of $0.38 \mathrm{~mm}$ in diameter, manipulated by reducing method when qi arrived. Heaven-penetrating cooling technique was used particularly for Qiuxu (GB 40). The needles were retained for $30 \mathrm{~min}$, manipulated every $5 \mathrm{~min}$ during retaining. The treatment was performed once everyday, 10 times denoting a treatment course.

\section{Results}

\subsection{Criteria of therapeutic effect}

Cured: Symptoms were gone thoroughly after treatment, no relapse in the follow-up study for more than half a year.

Improved: Symptoms were markedly reduced.

Failed: There was no remarkable improvement after treatment.

\subsection{Therapeutic result}

Of all the subjects, 54 were cured, 20 improved, 2 failed, and the total effective rate was $97.0 \%$.

\subsection{Case study}

A male patient, 19 years, student, visited on Sept 12, 1995 for the first time. He complained of nasal stuffiness, yellow turbid nasal discharge, with impaired sense of smell, accompanied by dizziness, headache, bitter taste, and hypochondriac pain. Greasy, yellow tongue coating and rapid wiry pulse were present. The patient was diagnosed with damp-heat in gallbladder and should be treated through clearing heat from gallbladder and unblocking the nasal passage. The aforementioned acupoints were selected for acupuncture therapy, and the patient was cured in 2 courses of treatment.

\section{Discussion}

Sinusitis is mainly manifested by turbid nasal discharge, nasal stuffiness and loss or declining of smell, also named "Nao Lou" in traditional Chinese medicine, which is categorized to be chronic sinusitis, acute or chronic paranasal sinusitis. Clinically, most cases are attributed to damp-heat in the liver and gallbladder going upwards affecting the nasal orifice. Hence, as the Yuan-Primary point of the Gallbladder Meridian, Qiuxu (GB 40) was selected as the major point for treating Sinusitis. Reducing method and Heaven-penetrating cooling technique are for clearing the retained heat. The physician should take into account that the needling sensation should be radiated to the affected area. Yingxiang (LI 20) and Fengchi (GB 20) assist to unblock the nasal passage and Yinlingquan (SP 9) to clear damp-heat and drain the nasal passage. The above acupoints work together to achieve a satisfactory therapeutic effect.

Translator: HONG Jue (洪玨) 